\title{
Experimental measurement of the isolated magnetic susceptibility
}

\author{
D. Billington $\odot,{ }^{1}$ C. Paulsen, ${ }^{2}$ E. Lhotel, ${ }^{2}$ J. Cannon, ${ }^{1}$ E. Riordan $\odot,{ }^{1}$ M. Salman, ${ }^{1}$ G. Klemencic, ${ }^{1}$ C. Cafolla-Ward, ${ }^{1}$ \\ D. Prabhakaran, ${ }^{3}$ S. R. Giblin $\odot,{ }^{1}$ and S. T. Bramwell ${ }^{4}$ \\ ${ }^{1}$ School of Physics and Astronomy, Cardiff University, Cardiff CF24 3AA, United Kingdom \\ ${ }^{2}$ Institut Néel, CNRS and Université Grenoble Alpes, 38000 Grenoble, France \\ ${ }^{3}$ Clarendon Laboratory, Physics Department, Oxford University, Oxford OX1 3PU, United Kingdom \\ ${ }^{4}$ London Centre for Nanotechnology and Department of Physics and Astronomy, University College London, \\ 17-19 Gordon Street, London WC1H OAH, United Kingdom
}

(Received 28 February 2021; accepted 1 June 2021; published 15 July 2021)

\begin{abstract}
The isolated susceptibility $\chi_{\mathrm{I}}$ may be defined as a (nonthermodynamic) average over the canonical ensemble, but while it has often been discussed in the literature, it has not been clearly measured. Here, we demonstrate an unambiguous measurement of $\chi_{\mathrm{I}}$ at avoided nuclear-electronic level crossings in a dilute spin ice system, containing well-separated holmium ions. We show that $\chi_{\mathrm{I}}$ quantifies the superposition of quasiclassical spin states at these points and is a direct measure of state concurrence and populations.
\end{abstract}

DOI: 10.1103/PhysRevB.104.014418

\section{INTRODUCTION}

Alternating current susceptometry $[1,2]$ is a traditional probe of magnetic response at applied frequencies $\omega /(2 \pi)$ of up to $10^{6} \mathrm{~Hz}$ [3]. As $\omega \rightarrow 0$, the isothermal susceptibility $\chi_{T}$ is measured, while, historically, there was much debate as to whether the high-frequency response could be approximated as a quasistatic adiabatic susceptibility $\chi_{S}$ [1] or a quasistatic isolated (or quantum adiabatic) susceptibility $\chi_{\mathrm{I}}$ [4-6]. The latter is a particularly interesting response function as it reveals aspects of a system that are not exposed by thermodynamic measurements, yet there do not seem to be any examples where $\chi_{\mathrm{I}}$ has been clearly observed [7]. Here, we demonstrate an experimental measurement of $\chi_{\mathrm{I}}$ at avoided level crossings in a simple spin system and show how it is a direct measure of the concurrence, or superposition of two quasiclassical spin states, and can be used to measure state populations.

The three susceptibilities, $\chi_{T}, \chi_{S}$, and $\chi_{\mathrm{I}}$, may be precisely defined (see the Appendix) with respect to canonical ensemble averages:

$$
\begin{gathered}
\chi_{T}=\frac{\partial M}{\partial H}=\frac{1}{V} \frac{\partial}{\partial H}\left(\sum_{i} m_{i} p_{i}\right), \\
\chi_{S}=\chi_{T}-\frac{\mu_{0} V T(\partial M / \partial T)^{2}}{C_{H}}, \\
\chi_{\mathrm{I}}=\frac{1}{V} \sum_{i}\left(\frac{\partial m_{i}}{\partial H}\right) p_{i},
\end{gathered}
$$

where the sum is over eigenstates $i$ of the Hamiltonian (which are not generally simple spin states), $H$ is the applied field, $M$ is the equilibrium magnetization, $V$ is the volume, $T$ is the temperature, $m_{i}=-\partial E_{i} / \partial B$ (with $B=\mu_{0} H$ ) is the magnetic moment of eigenstate $i, C_{H}$ is the magnetic heat capacity at constant applied field, $p_{i}=e^{-E_{i} / k_{\mathrm{B}} T} / Q$ is a Boltzmann population, and $Q=\sum_{i} e^{-E_{i} / k_{\mathrm{B}} T}$ is the partition function, with $k_{\mathrm{B}}$ being Boltzmann's constant. Note that the quantum adiabatic susceptibility $\chi_{\mathrm{I}}$ is not necessarily equal to the thermodynamic adiabatic susceptibility $\chi_{s}$. Also, $\chi_{\mathrm{I}}$ cannot be expressed as a second derivative of the free energy, so it is not a thermodynamic property. It has been proved that $\chi_{T} \geqslant \chi_{S} \geqslant$ $\chi_{\mathrm{I}} \geqslant 0[5]$.

In experiment, the frequency-dependent susceptibility $\chi(\omega)$ generally measures $\partial M / \partial H=\chi_{T}$ as $\omega \rightarrow 0$. A purely real response, $\chi(\omega)=\chi^{\prime}(\omega)$, at high frequency could equate to $\chi_{S}$ if the only effect of finite frequency is to decouple the system from the heat bath [1], or it could equate to $\chi_{\mathrm{I}}$ if the state populations of the system remain equal to those that existed before the field perturbation was applied. In the latter case, if the fixed values $p_{i}$ are not equilibrium populations for all $H(t)$, then the response of the system is nonergodic. The experiment measures a time $t$ average, $\partial M(t) / \partial H(t)$, that is equal to $\chi_{\mathrm{I}}$ but is not equal to the ensemble-averaged $\partial M / \partial H=\chi_{T}$. However, $\chi_{\mathrm{I}}$ can still be calculated by a different average over the canonical ensemble, as given in Eq. (3).

To see how this may come about in practice, in Fig. 1(a) we describe an idealized spin system where the driving period $\tau=2 \pi / \omega$ is compared to well-separated spin-lattice $\left(\tau_{1}\right)$ and spin-spin $\left(\tau_{2} \ll \tau_{1}\right)$ relaxation times [8]. At very low frequency, the magnetic system will remain in thermal equilibrium throughout the field cycle, giving $\chi_{T}$ as the real response. As $\omega$ is increased until $\tau \ll \tau_{1}$, equilibrium with the lattice and heat bath is lost, but spin-spin interactions retain thermal equilibrium between spins, giving an adiabatic response, $\chi(\omega)=\chi_{S}$ [1]. If the drive frequency is further increased until $\tau \ll \tau_{2}$, then equilibrium between spins is lost, and the perturbing field acts on the state populations that existed before the perturbation was applied, so $\chi(\omega)=\chi_{\mathrm{I}}$. Hence, with $\tau_{1}$ and $\tau_{2}$ well defined and well separated, the susceptibility $\chi(\omega)$ takes the form of a series of decreasing 


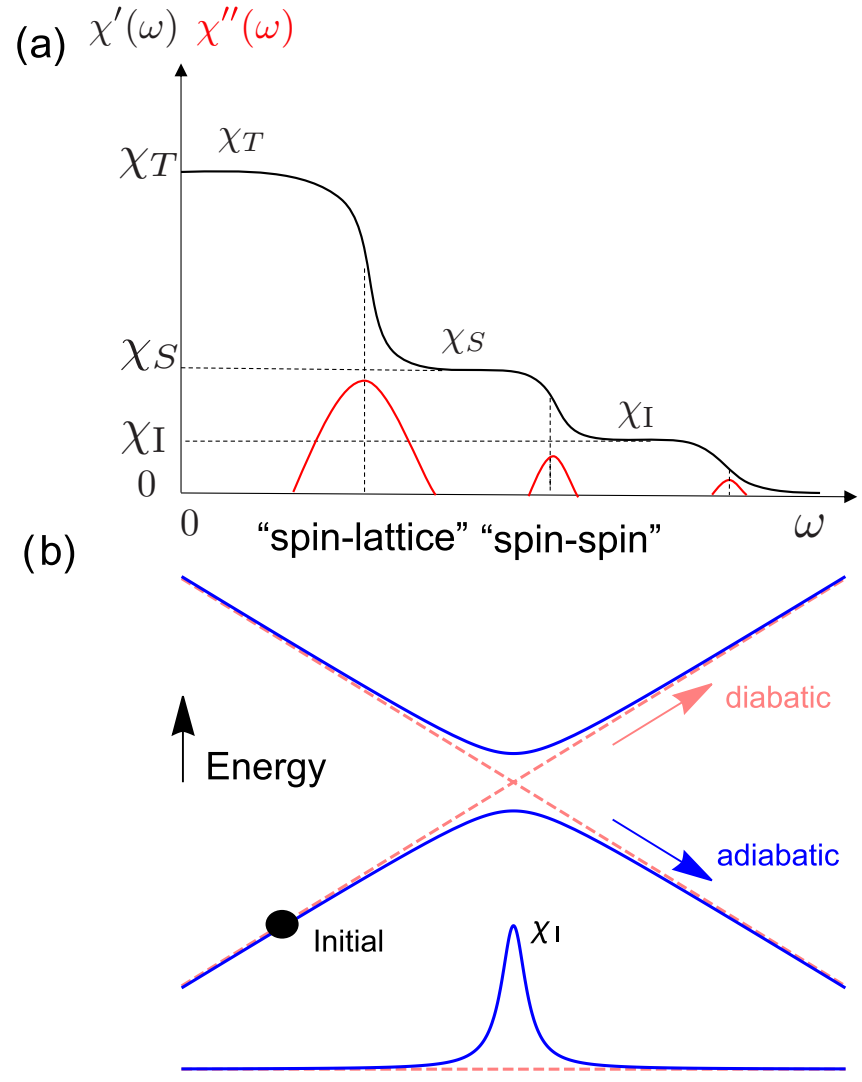

Field

FIG. 1. (a) Schematic showing the frequency-dependent decoupling of the real (black) and imaginary (red) parts of the magnetic susceptibility in a system with well-separated spin-lattice and spinspin relaxation times. One can expect three plateaus of purely real response corresponding to $\chi_{T}, \chi_{S}$, and $\chi_{\mathrm{I}}$, respectively. (b) Avoided level crossing (upper blue curves). In this paper we are solely interested in adiabatic evolution (blue). It is illustrated for an initial state on the lower branch, as indicated by a black circle. Also illustrated is diabatic evolution (pink) that could occur if there were a Landau-Zener crossover at frequencies greater than those studied here. The lower curves (same color code) indicate the corresponding isolated susceptibilities for the ensemble of two state systems treated in Sec. II, where the adiabatic case yields a peak in $\chi_{\mathrm{I}}$, while the diabatic case gives zero, as shown in (a).

plateaus [Fig. 1(a)], reminiscent of a dielectric response. As $\omega$ is increased, the susceptibility on each plateau- $\chi_{T}, \chi_{S}$, and $\chi_{\mathrm{I}}$, respectively — can be calculated in a quasistatic limit [Eqs. (1)-(3)].

In this paper, we will be particularly interested in avoided level crossings [Fig. 1(b)] where the curvature of the state energies with field allows a finite $\chi_{\mathrm{I}}$ according to Eq. (3). In this context, it is important to stress that the isolated response is, by definition, adiabatic in the quantum mechanical sense [9]. The Landau-Zener effect [10] (a dynamical effect) is therefore not relevant to this paper except insofar as it could imply a further crossover, with increasing frequency, from the case where the crossing is traversed adiabatically to the case where it is traversed diabatically [see Fig. 1(b)]. In view of the subsequent discussion, this would drive the susceptibility to zero, a feature that we have illustrated in Fig. 1(a). An experimental example of a Landau-Zener crossover in a rare-earth complex is given in Ref. [11].

The response of real magnetic systems can be far more complicated than implied by the simplified picture of Fig. 1(a), but there will always be a gradual crossover, with increasing frequency, from a scenario in which state populations change on the timescale of the field cycle to one in which they do not. The low-frequency regime can be treated by a master equation approach (see, for example, Refs. [6,12]), which accounts for state population changes, while the high-frequency regime can be treated by a Kubotype linear response approach [13], which assumes fixed populations. Indeed, neutron scattering, which probes the response at relatively high frequencies, is very successfully treated by the latter approach [14].

In contrast to $\chi_{T}$, which irretrievably mixes field-induced changes in quantum states with field-induced changes in state populations, $\chi_{\mathrm{I}}$ may be viewed as a more direct measure of quantum spectra. It is, further, a general measure of the superposition of quasiclassical spin states. Thus, if an energy eigenstate is, say, a pure "spin-up" state, it will have zero isolated susceptibility because the magnetic moment is field independent [see Eq. (3)]. A finite $\chi_{\mathrm{I}}$ can, however, be observed if spin up and spin down are superposed, which allows the magnetic moment to evolve with field. For example, in the case that zero-field states may be pure spin up/spin down or admixtures thereof, application of the Zeeman operator as a perturbation gives $\chi_{\mathrm{I}}$ in the form [6]

$$
\chi_{\mathrm{I}}=\mu_{0}(V Q)^{-1} \sum_{i, j} \frac{\left.e^{-\beta E_{i}}-e^{-\beta E_{j}} \neq E_{i}\right)}{E_{j}-E_{i}}|\langle i|\hat{\mu}| j\rangle|^{2},
$$

where $\hat{\mu}$ is the magnetic moment operator and $\beta=1 / k_{\mathrm{B}} T$. This contains finite matrix elements only if different energy eigenstates $i, j$ contain both spin-up and spin-down components, which occurs at avoided level crossings, for example [15].

In this paper, we demonstrate a particularly simple magnetic system in which the isolated susceptibility can be measured and analyzed. The paper first considers relevant theory and then describes our experimental results. In Sec. II we solve the statistical mechanics of a simple two-state paramagnetic system with an idealized spin Hamiltonian, designed to emphasize differences between the three susceptibilities [Eqs. (1)-(3)] and to highlight the connection between isolated susceptibility and state concurrence. Section III shows how a variant of this Hamiltonian may be realized by approximating the hyperfine Hamiltonian of dilute spin ice. In particular, a strong isolated response and perfect state concurrence at avoided level crossings in finite field are predicted. Section IV then describes our experiments that confirm these predictions at $T \geqslant 2 \mathrm{~K}$, where the system is prepared in a state of thermal equilibrium. Section $\mathrm{V}$ describes analogous low-temperature experiments, down to $T=76 \mathrm{mK}$, that reveal how, when the system cannot be brought to equilibrium, isolated susceptibility can be used as a sensitive probe of the nonequilibrium state populations. Conclusions are subsequently drawn in Sec. VI. 


\section{A TWO-STATE SYSTEM}

We start by considering a simple two-state system in which the difference between the three susceptibilities of Eqs. (1)(3) may be made explicit. The system consists of an ensemble of noninteracting spins, each with $S=1 / 2$ and Hamiltonian

$$
\hat{H}=2 \mu B \hat{S}^{z}+2 \Delta \hat{S}^{x} .
$$

Here, $\mu$ is the size of the magnetic moment of the pure spin-up or -down states, $\Delta$ is a perturbation, and the spin $\hat{S}$ operators are defined to be dimensionless. The first term in Eq. (5) is the Zeeman interaction, and the second term mixes magnetic (spin-up and spin-down) states. This Hamiltonian is easily diagonalized, with eigenvalues characteristic of an avoided level crossing at $B=0$ [see Fig. 1(b), blue lines],

$$
E_{ \pm}= \pm \mathcal{E}, \quad \mathcal{E}=\sqrt{(\mu B)^{2}+\Delta^{2}},
$$

and eigenstates

$$
\left|\psi_{ \pm}\right\rangle=\frac{\Delta|\uparrow\rangle \pm(\mathcal{E} \mp \mu B)|\downarrow\rangle}{\sqrt{( \pm \mathcal{E}-\mu B)^{2}+\Delta^{2}}}
$$

characteristic of a gradual superposition of spin up and spin down as the crossing is approached. The two quasiclassical states are completely superposed at the avoided level crossing in zero field but are not superposed far from that point.

To characterize this behavior quantitatively, following Ref. [16], we may introduce the "concurrence" $\mathcal{C}$ of a state $|\psi\rangle$ as its overlap with its spin-reversed equivalent $|\tilde{\psi}\rangle$ :

$$
\mathcal{C}(\psi)=|\langle\psi \mid \tilde{\psi}\rangle| .
$$

This takes the value $\mathcal{C}=0$ for the nonsuperposed states $|\uparrow\rangle$ and $|\downarrow\rangle$, which are the eigenstates corresponding to $E_{ \pm}$in sufficiently strong fields $|B|$, and $\mathcal{C}=1$ for the fully superposed states $\psi_{ \pm}=(|\uparrow\rangle \pm|\downarrow\rangle) / \sqrt{2}$, which are the eigenstates at the zero-field avoided level crossing. It is then easy to show that the isolated susceptibility is a direct measure of concurrence as a function of field $\mathcal{C}(B)$, given by

$$
\chi_{\mathrm{I}}=\frac{\mu_{0}}{V} \frac{\mu^{2}}{\Delta} \sum_{ \pm} \mp p_{ \pm} \mathcal{C}^{3},
$$

where $\mathcal{C}(B)=\Delta / \sqrt{\Delta^{2}+B^{2} \mu^{2}}$ and $p_{ \pm}$represent the normalized state populations. Using Boltzmann probabilities, this becomes

$$
\chi_{\mathrm{I}}=\frac{\mu_{0}}{V} \frac{\mu^{2} \mathcal{C}^{3}}{\Delta} \tanh \left(\frac{\beta \Delta}{\mathcal{C}}\right) \approx \mu_{0} V^{-1} \beta \mu^{2} \mathcal{C}(B)^{2},
$$

where the right-hand approximation is valid in the hightemperature limit. The isolated susceptibility is then a Lorentzian function of field:

$$
\chi_{\mathrm{I}}=\frac{\mu_{0} \mu^{2}}{V k_{\mathrm{B}} T} \frac{1}{1+\left(\mu^{2} / \Delta^{2}\right) B^{2}} .
$$

With knowledge of the exact eigenstate energies as a function of field, all three susceptibilities in Eqs. (1)-(3) can therefore be calculated. The isolated response is a maximum at $B=0$ when $\mathcal{C}=1$ and $\chi_{\mathrm{I}}(0)=\chi_{S}(0)=\chi_{T}(0)=$ $\mu_{0} V^{-1} \beta \mu^{2}$. The closely spaced eigenstates of the system with energy $E_{ \pm}= \pm \Delta$ have precisely zero magnetic moment at the avoided crossing in zero field, gaining a moment only through their mixing by the second-order Zeeman effect as the field is applied. However, with increasing field, the three susceptibilities diminish at different rates, such that $\mu_{0} V^{-1} \beta \mu^{2}>$ $\chi_{T}(B)>\chi_{S}(B)>\chi_{\mathrm{I}}(B)$.

The more complex Hamiltonian considered subsequently will retain many of the characteristics of this simple example, though some important differences of detail arise from the fact that, in the more complex case, the avoided level crossings occur at finite applied field.

\section{HYPERFINE HAMILTONIAN}

A model Hamiltonian similar to that considered in Sec. II may be realized experimentally in the dilute limit of an Isinglike spin system, such as very dilute samples of spin ice, $\mathrm{Y}_{1.9975} \mathrm{Ho}_{0.0025} \mathrm{Ti}_{2} \mathrm{O}_{7}$, studied here. Figure 2(a) illustrates the spin ice geometry where every fourth (apical) spin is parallel or antiparallel to the field, while the other three (basal) spins have only a small parallel or antiparallel component. We will be interested mainly in the response of the apical spin, but the three basal spins also need to be considered as they provide an important correction.

The non-Kramers holmium $\left(\mathrm{Ho}^{3+}\right)$ ion with nuclear spin $I=7 / 2$ would be expected to afford a weakly "split" electronic spin doublet with effective spin $S=1 / 2$ [17] and $z=$ $\langle 111\rangle$ quantization axis ensured by the large trigonal crystal field [18]. For an ideal non-Kramers doublet of this sort, neglecting the quadrupole term, the hyperfine Hamiltonian may be written as

$$
H_{\text {hyper }}=2 A \hat{S}^{z} \hat{I}^{z}, \quad 2 A=g_{\|}\left(\frac{A_{J}}{g_{J}}\right),
$$

where $A_{J} / g_{J}$ should be essentially the same for all Ho salts (note that Ho has a single isotope). For $\mathrm{Ho}^{3+}$ in the spin ice environment, the parallel $g$ factor is $g_{\|} \approx 19$ (see below), and the hyperfine parameter is $A / k_{\mathrm{B}} \approx 0.3 \mathrm{~K}$. If higher states are relevant, they can induce a further transverse term $2 A^{\prime}\left(\hat{S}^{x} \hat{I}^{x}+\right.$ $\left.\hat{S}^{y} \hat{I}^{y}\right)$, with $2 A^{\prime}=g_{\perp}\left(\frac{A_{J}}{g_{J}}\right)$, but here, any perpendicular $g$ factor $g_{\perp}$ is certainly extremely small [18] and is henceforth assumed to be zero. More importantly, Abragam and Bleaney [17] recommend the addition of a term $2 \Delta\left(\hat{S}^{x}+\hat{S}^{y}\right)$ with a distribution of parameters $\Delta$ arising from local strains [19]. We can suppress the $y$ term without loss of generality, and including the Zeeman term for the field $B$ applied parallel to [111], the effective Hamiltonian for the apical spins becomes

$$
\hat{H}_{\text {apical }}=2 A \hat{S}^{z} \hat{I}^{z}+2 \mu B \hat{S}^{z}+2 \Delta \hat{S}^{x},
$$

where $\mu=(1 / 2) g_{\|} \mu_{\mathrm{B}} \approx 10 \mu_{\mathrm{B}}$. A similar Hamiltonian may be constructed for the basal spins:

$$
\hat{H}_{\text {basal }}=2 A \hat{S}^{z} \hat{I}^{z}+2(\mu / 3) B \hat{S}^{z}+2 \Delta \hat{S}^{x} .
$$

The factor $(1 / 3)$ in this equation arises from the angle $\arccos (1 / 3)$ that the basal spins subtend with the applied field. Note also that there is no direct coupling of the field with $\hat{S}^{x}$ because $g_{\perp}=0$.

Taking $\hat{H}_{\text {apical }}$ as an example, these Hamiltonians may be represented in the basis of states $\left|m_{S}\right\rangle\left|m_{I}\right\rangle$, where the only offdiagonal terms are those with $m_{I}-m_{I}^{\prime}=0$ and $m_{S}-m_{S}^{\prime}=$ \pm 1 . Here, $m_{S}= \pm 1 / 2$, and $m_{I}= \pm 7 / 2, \pm 5 / 2, \pm 3 / 2, \pm 1 / 2$. The Hamiltonian can therefore be separated into a series of 

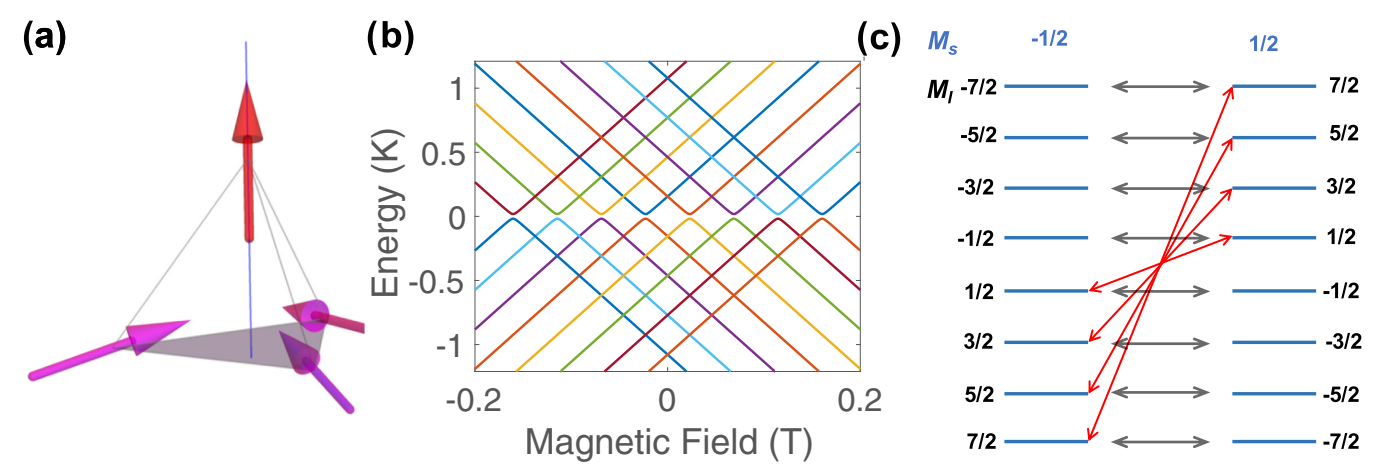

FIG. 2. (a) The four Ising-like $\langle 111\rangle$ spin orientations of $\mathrm{Ho}^{3+}$ with respect to the [111] direction of the applied field (blue) and the basal plane (shaded; apical spin is in red, basal spins are in magenta). In the dilute sample only one or zero magnetic sites are likely to be occupied by magnetic $\mathrm{Ho}^{3+}$ in any given tetrahedron of the crystal structure. (b) Energy diagram of the hyperfine levels of an apical Ho ${ }^{3+}$ ion as a function of magnetic field with an effective splitting of $\Delta / k_{\mathrm{B}}=0.013 \mathrm{~K}$, showing direct and avoided level crossings. (c) The effective electronic spin- $1 / 2 \mathrm{Ho}^{3+}$ ion has eight Zeeman split levels which are degenerate in zero field (gray arrows). As the field is increased from zero to positive values, there are four level crossings where only the electron spin reverses. When brought into resonance by applied field, superposed $\pm m_{S}$ states have finite isolated susceptibility.

$2 \times 2$ blocks of the type

$$
\hat{H}^{\prime}=\left[\begin{array}{cc}
A m_{I}+\mu B & \Delta \\
\Delta & -A m_{I}-\mu B
\end{array}\right]
$$

one for each value of $m_{I}$. The Hamiltonian is thus easily diagonalized, as above, with eigenvalues

$$
E_{m_{I}, \pm}= \pm \sqrt{\left(A m_{I}+\mu B\right)^{2}+\Delta^{2}} .
$$

Hence, energies and magnetic moments of the Hamiltonian (15) map to those of Eqs. (5)-(7), with $\mu B$ replaced by $A m_{I}+\mu B$ : the nuclear spin acts as an effective field that adds to the applied field. It is then straightforward to evaluate the partition function corresponding to Eq. (16) and then derive the susceptibilities using Eqs. (1)-(3) with the magnetic moment defined as $m_{i}=-\partial E_{i} / \partial B$.

The consequent energy diagram for the apical spins as a function of magnetic field is shown in Fig. 2(b), illustrating the field-dependent direct and avoided level crossings. There are two degenerate ladders of energy levels, each corresponding to $m_{S}= \pm 1 / 2$, with $m_{I}$ defining the rungs of the ladder, as depicted in Fig. 2(c). Starting from the zero field as depicted in Fig. 2(c), as a magnetic field is applied, neglecting its weak coupling with the nuclear moment, the two sets of states shift by the electronic first-order Zeeman energy difference. Equivalent level crossings occur in both positive and negative fields: in the following we enumerate the crossings with respect to only positive fields. For such positive fields, there are, in principle, eight values of the applied field where energy level crossings occur. However, the isolated susceptibility $\chi_{\mathrm{I}}(H)$ strongly peaks only at the subset of four avoided level crossings where the nuclear spin state does not change [see Fig. 2(c)]. At these points, the up and down electronic spin states, $|\uparrow\rangle$ and $|\downarrow\rangle$, respectively, are fully superposed and show complete concurrence, $\mathcal{C}=1$, while off resonance, the spin states tend towards simple spin up or down with $\mathcal{C}=0$ [see Eqs. (7) and (8)]. However, in contrast to the simpler case considered above, even at the special points of resonance, $\chi_{\mathrm{I}}$ is suppressed by a significant factor with respect to $\chi_{T}$ (see Fig. 3). Physically, the reason is that, at a given avoided crossing, the isolated susceptibility is finite only for electronic states associated with a single nuclear spin state, a small fraction $[\sim 1 /(2 I+1)$ at high temperature] of the total available. One might say that the field-driven system is ergodic for one nuclear spin state but nonergodic for the rest, in contrast to the simpler case of Eq. (9), where the system is fully ergodic at the $B=0$ resonance and $\chi_{T}=\chi_{\mathrm{I}}$ as envisaged by Kubo [13].

Considering just the isolated susceptibility, we refer to Fig. 3 and label the four isolated susceptibility peaks (from left to right) as 1-4. A similar calculation (not shown) may be carried out for the basal spin Hamiltonian, Eq. (14). The basal spins are found to contribute a small peak coincident with 2 and another small peak at three times that field (to the right of 4 in Fig. 3). The reason these features are relatively small is that the susceptibility scales as the moment squared and the projected moment of the basal spins is $1 / 3$ that of the apical ones, but they have three times the population, so the peaks are only one third $\left[=3 \times(1 / 3)^{2}\right]$ the height of the apical spin peaks. The basal spins therefore represent a small correction

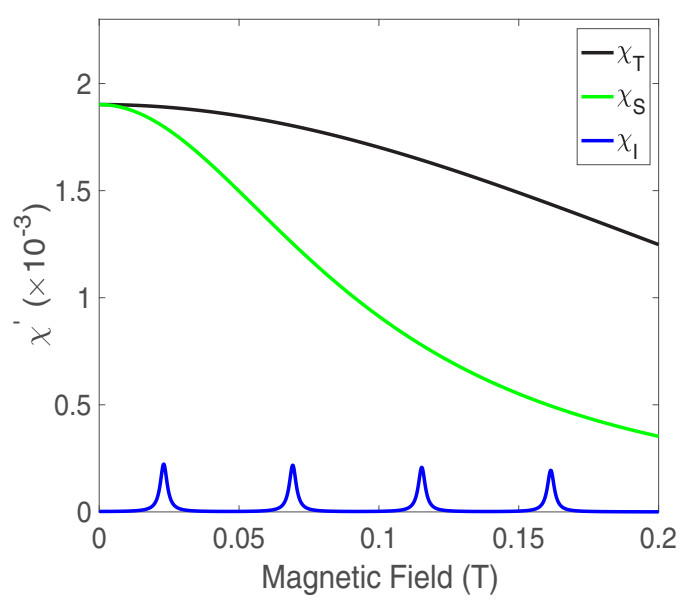

FIG. 3. Calculated values of the three susceptibilities [Eqs. (1)(3)] at $T=2.1 \mathrm{~K}$ for the apical spin Hamiltonian (13), with spin concentration equal to that of the sample studied. 


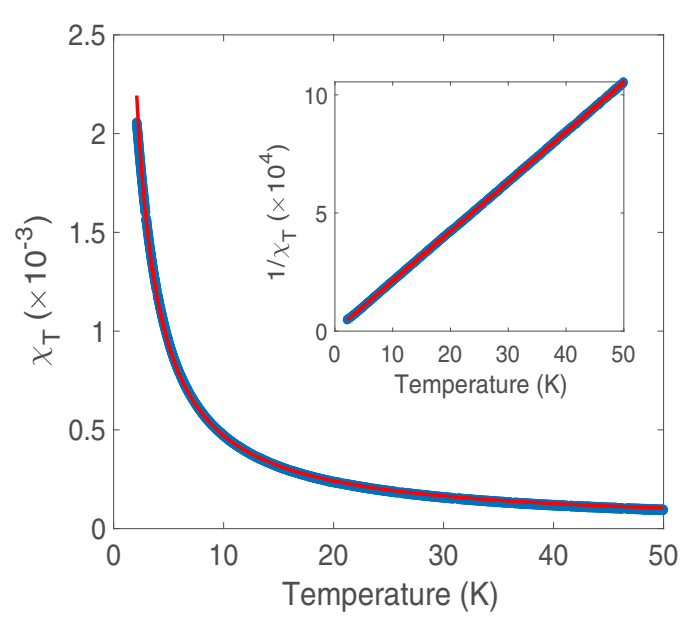

FIG. 4. Temperature dependence of the dc field-cooled susceptibility of $\mathrm{Y}_{1.9975} \mathrm{Ho}_{0.0025} \mathrm{Ti}_{2} \mathrm{O}_{7}$ measured along the [111] axis (blue points) compared to a Curie law fit summed with a small temperature-independent component (red line). Inset: graph replotted as $1 / \chi_{T}$.

to peak 2 and a small rising background correction to the right of peak 4 .

More generally, a strongly structured nuclear-electronic response is, indeed, a long-established behavior [20] of dilute $\mathrm{Ho}^{3+}$ ions in crystals, which have been impressively analyzed with master-equation-based approaches in several works [21-25]. In general, various transitions are possible depending upon coupling with the local environment of the effective spins, which generates a combination of intrinsic and interaction-induced direct and avoided level crossings, respectively. Our Hamiltonian, Eq. (15), describes a highly simplified limiting case where the response is mapped on to the quasistatic isolated susceptibility, Eq. (3).

\section{EXPERIMENT}

A single crystal of $\mathrm{Y}_{1.9975} \mathrm{Ho}_{0.0025} \mathrm{Ti}_{2} \mathrm{O}_{7}$ was grown by the optical floating-zone technique [26]. It was aligned with the applied field along the cubic [111] axis. Our ac and dc susceptibility measurements were made at $T \geqslant 2 \mathrm{~K}$ using a Quantum Design physical property measurement system and at $T<2 \mathrm{~K}$ using a low-temperature superconducting quantum interference device magnetometer developed at the Institut Néel, Grenoble [27].

To confirm the stoichiometry $x$ and the paramagnetic approximation, the bulk susceptibility was measured after cooling in a field of $0.1 \mathrm{~T}$. The data were fitted to the sum of a Curie law $\chi_{T}=C / T$ and a very small temperatureindependent component. The Curie constant $C$ was specified for holmium concentration $x=0.0025$, while the $g$ factor $g_{\|}$ entering into the theoretical expression for $C$ [28] was treated as a fitting parameter. This gave $g_{\|}=19.0$, which we use in subsequent analysis. The excellent fit shown in Fig. 4 confirms that the nominal $x=0.0025$ is accurate. A splitting between field-cooled and zero-field-cooled susceptibilities (not shown) was observed below $T=3.6 \mathrm{~K}$. This shows that the spins are already falling out of equilibrium on the timescale of this

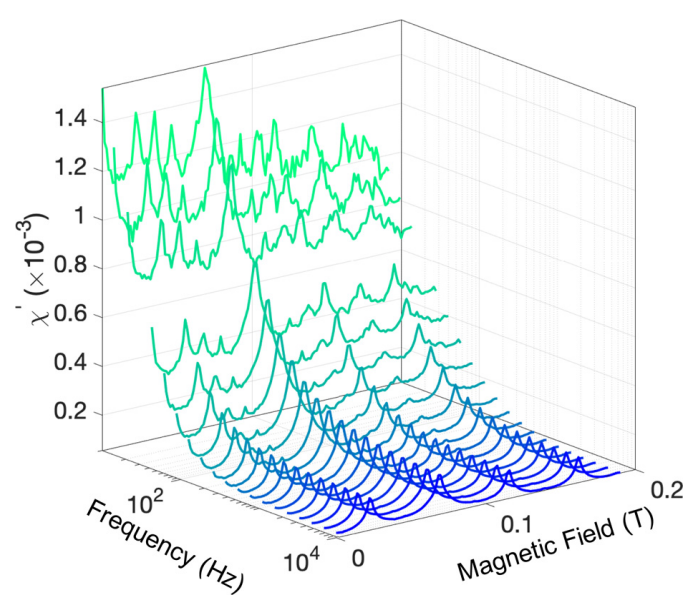

FIG. 5. Frequency and magnetic field dependence of the real part of the susceptibility measured at $2.1 \mathrm{~K}$ with an ac field amplitude of $0.2 \mathrm{mT}$, demonstrating a gradual reduction to four peaks as the frequency is increased.

"static" measurement. It is consistent with our observations of the frequency-dependent susceptibility, as described below.

The ac susceptibility $\chi^{\prime}(\omega)$ measured at $2.1 \mathrm{~K}$ (with a probe field of $0.2 \mathrm{mT}$ ) is shown in Fig. 5. At low frequency and zero field $\chi^{\prime}(\omega)$ approaches $\chi_{T}$ of Fig. 4, and while it still falls short by some $25 \%$ at the lowest applied frequency, the $\chi_{T}$ plateau in Fig. 1(a) can safely be presumed to exist at frequencies lower than those applied here. In finite field, multiple peaks evolve with increasing frequency to become four distinct peaks by $10 \mathrm{kHz}$. The spectrum and amplitude of these four peaks correspond closely to the isolated susceptibility of Fig. 3, and we may therefore identify the distinctive plateau in their frequency evolution with the $\chi_{\mathrm{I}}$ plateau in Fig. 1(a). As anticipated, the high-frequency susceptibility on the peaks is essentially a real response, with any imaginary component being only a few percent of the real part [see Fig. 6(a)]. At intermediate frequencies, the $\chi_{S}$ plateau in Fig. 1(a) is not clearly resolved at any value of applied field, presumably because spin-spin and spin-lattice times are not sharply defined or separated in this system. The multipeak structure in the intermediate frequency range is similar to that described for other Ho systems [22].

It is clear from Fig. 5 that transitions at high frequency are of the type $|1 / 2\rangle\left|m_{I}\right\rangle \rightarrow|-1 / 2\rangle\left|m_{I}\right\rangle$, consistent with our effective Hamiltonian for spins parallel to the field. Also, as well as showing the four peaks associated with the apical spins, the experimental spectra evidence the expected additional small component on peak 2 from the basal spins: that is, peak 2 is approximately $1 / 3$ higher than the other peaks. There is also what seems, at first sight, like an unexpected "nonzero background" that fills in the gaps between the peaks. However, the fact that it is mainly real [see Fig. 6(a)] identifies it as part of the isolated response itself.

We now consider how these data can be fitted quantitatively. The theoretical isolated susceptibility is found by summing the susceptibilities $\chi_{\mathrm{I}}$ of the apical and basal spins calculated as described above, using Eq. (3), with Boltzmann probabilities for the nuclear-electronic spin states and the 

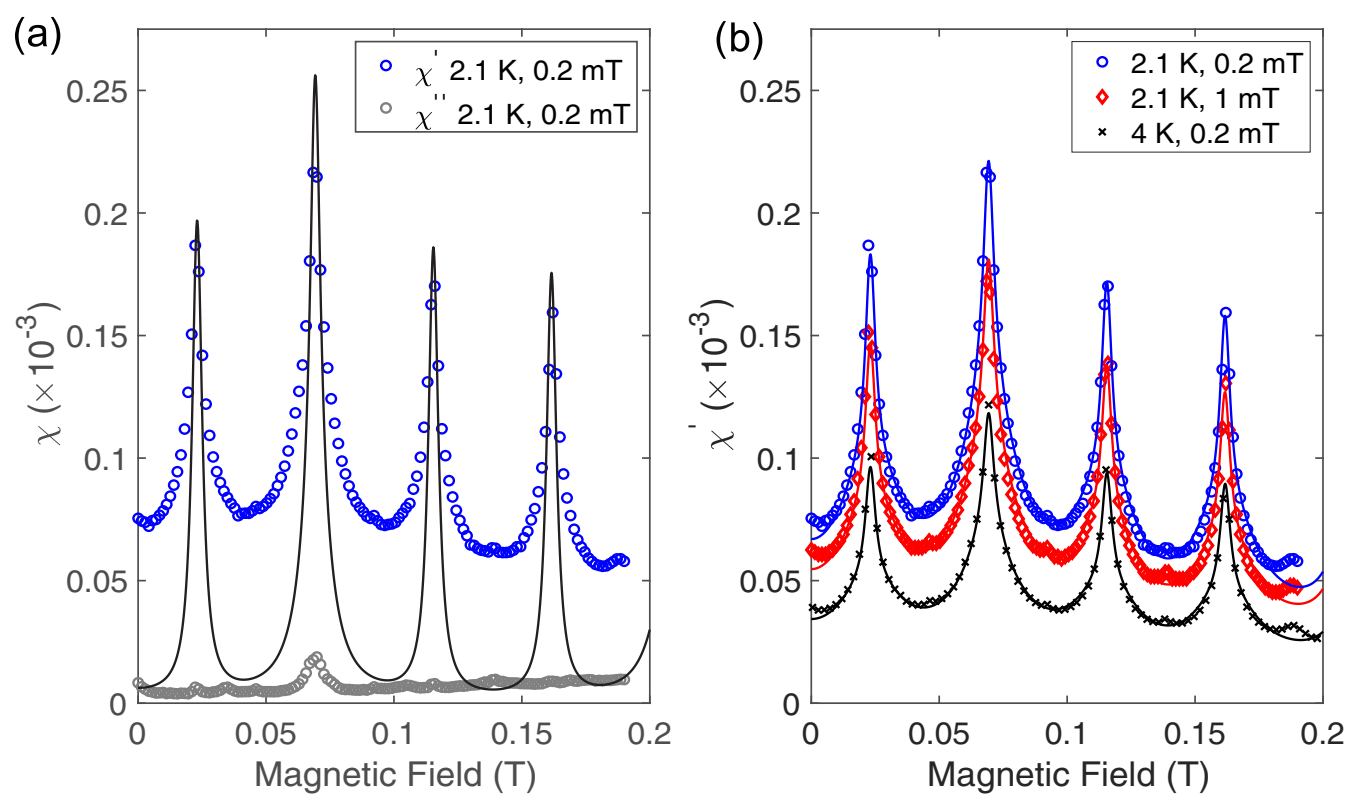

FIG. 6. (a) The real (blue points) and imaginary (gray points) part of the measured susceptibility at $2.1 \mathrm{~K}, 10 \mathrm{kHz}$, and a probe field of $0.2 \mathrm{mT}$ compared to the theory, with a single value of $\Delta / k_{\mathrm{B}}=0.015 \mathrm{~K}$ (black line). (b) Experiment versus theory with an empirical distribution of $\Delta$ [Eq. (18)]. The fitted parameters are $f_{1}=0.511(6), f_{2}=0.352(3)$ for $2.1 \mathrm{~K}, 0.2 \mathrm{mT} ; f_{1}=0.455(6), f_{2}=0.312(3)$ for $2.1 \mathrm{~K}, 1.0 \mathrm{mT}$; and $f_{1}=0.52(1), f_{2}=0.335(4)$ for $4 \mathrm{~K}, 0.2 \mathrm{mT}$. These parameters affect the peak shape, not peak heights, and are expected to evolve with probe field but not significantly with temperature. It is also possible to fit the data with smooth distributions (see text).

hyperfine parameter refined to $A / k_{\mathrm{B}}=0.2945 \mathrm{~K}$ by fitting the experimental peak positions.

With this, the fits have no adjustable parameters except those connected with the distribution of $\Delta$, which can be estimated only empirically [17]. Figure 6(a) compares the experimental data with a single value of $\Delta / k_{\mathrm{B}}=0.015 \mathrm{~K}$. The peaks are quite well described, but the regions between them are underestimated. The broadened bases of the peaks suggest a contribution to the distribution from $\Delta / k_{\mathrm{B}} \approx 0.1 \mathrm{~K}$. There is also the possibility of some ions experiencing a very small $\Delta$, which raises a complication. For sufficiently small values of $\Delta$, the apparent response in an ac susceptibility experiment with finite probe field will approach zero because of the nonlinearity (and eventual saturation) of the magnetic moment with field. Our experiment used a probe field of $0.2 \mathrm{mT}$ from which we can estimate $\Delta / k_{\mathrm{B}}<0.002 \mathrm{~K}$ as the point beyond which the response will be suppressed.

To capture these properties in an empirical, parameterized, distribution, we consider one consisting of three $\delta$ functions (one at zero and one each at the lower and upper values of $\Delta$ discussed above):

$$
P(\Delta)=f_{0} \delta(\Delta)+f_{1} \delta(\Delta-0.015 \mathrm{~K})+f_{2} \delta(\Delta-0.1 \mathrm{~K})
$$

where we have suppressed factors of Boltzmann's constant for clarity. The $\delta$ function at zero does not contribute any response but is relevant through the normalization condition on the frequencies: $\sum_{i} f_{i}=1$. With this, we have two independent parameters to fit the data: $f_{1}$ and $f_{2}$.

Figure 6(b) compares fits to the data taken either at the same temperature $(2.1 \mathrm{~K})$ with a larger probe field $(1 \mathrm{mT})$ or at a larger temperature $(4 \mathrm{~K})$ with the same probe field $(0.2 \mathrm{mT})$. For the larger probe field, as would be anticipated, the "in- visible" part of the response (represented by $f_{0}$ ) is increased as more of the nonlinear response is sampled over the field cycle. As expected, the susceptibility derived at $1 \mathrm{mT}$ falls below that of the $0.2 \mathrm{mT}$ measurement, and $f_{0}=1-f_{1}-f_{2}$ derived from the fit was found to increase accordingly from 0.13 at $0.2 \mathrm{mT}$ to 0.23 at $1 \mathrm{mT}$. Changing temperature, on the other hand, yields parameters in close agreement with those found at $T=2 \mathrm{~K}$, again as expected.

Referring to Fig. 6(b), the apical response is clearly very well fitted by the model Hamiltonian. The basal response is slightly less well described, as a small imaginary component is detectable on peak 2. However, this part of the response is far too small to warrant introducing extra parameters into the model. The fact that quite an accurate fit is already obtained using a simple empirical distribution of $\Delta$ points to the conclusion that the model is essentially correct.

It is worth noting, however, that the empirical probability density for $\Delta$ is not uniquely specified by the data. To find alternatives to Eq. (17), tests were made with continuous distributions (represented mathematically as arrays of $\delta$ functions), and it was clear that some of these would return line shapes very similar to that generated by Eq. (17). The successful functions were typically peaked at $\Delta / k_{\mathrm{B}} \sim 0.01 \mathrm{~K}$ or less, with a "heavy" tail reaching to $0.1 \mathrm{~K}$ or beyond. For example, the simple function $P \sim 1 / \Delta$, with cutoffs at $\Delta / k_{\mathrm{B}}=0.0025,0.25 \mathrm{~K}$, gives a result very close to that of Eq. (17). In a non-Kramers ion like $\mathrm{Ho}^{3+}$, the distribution of $\Delta$ can arise, for example, from random strains or electric fields, combining with the Jahn-Teller effect [17]. Early experimental and theoretical work [29] has understood many of the mechanisms involved and the resulting distributions, but positively identifying the distribution appropriate for the present 


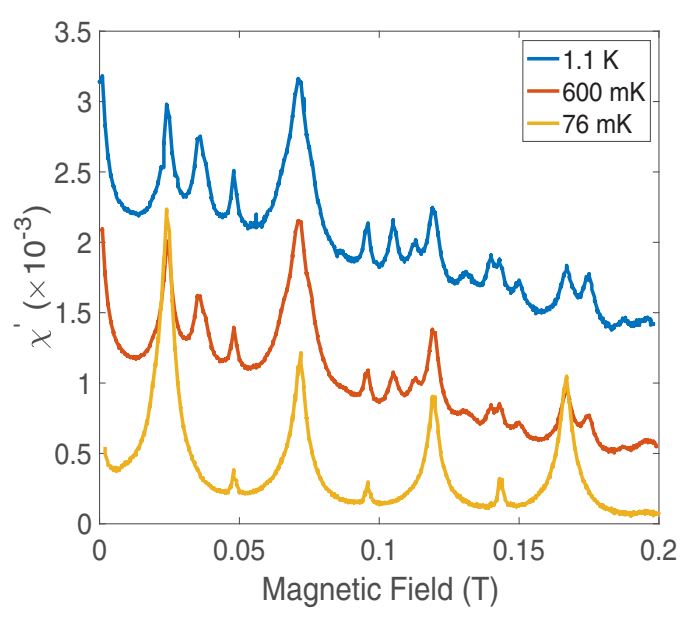

FIG. 7. The real part of the susceptibility at $11 \mathrm{~Hz}$ as a function of field and decreasing temperature, demonstrating that the isolated response is revealed by low temperature as well as high frequency and that the distribution of peak intensities is no longer that of a Boltzmann distribution (contrast with Fig. 6).

case would be a significant project (albeit an interesting one) that we defer to the future.

\section{LOW-TEMPERATURE BEHAVIOR}

Because the isolated susceptibility separates population changes from state vector changes, the decline of peak intensity with increasing field (having allowed for the basal spin contribution on peak 2) is a measure of the decline in population with increasing energy and hence a measure of the temperature. This gives an interesting method of directly investigating the effective temperatures reached when spin ice falls out of thermal equilibrium at $T<0.6 \mathrm{~K}$ [30]. Figure 7 shows the temperature dependence of $\chi^{\prime}$ at a fixed frequency of $11 \mathrm{~Hz}$ as the dilute sample is cooled. As the temperature is lowered to the base temperature of $76 \mathrm{mK}$, the four peaks indicative of isolated response again appear, but there are two features that mark these as reflecting nonequilibrium populations. First, at $76 \mathrm{mK}$, direct calculation shows that all peaks should have zero intensity if equilibrium is maintained, yet their observed intensities suggest a temperature of order $1 \mathrm{~K}$. Second, peak 4 is now anomalously intense, which is a signature that the system, initially zero field cooled, does not fully reequilibrate as the field is applied. Thus, referring to the energy level diagram in Fig. 2(b), if the system retains the equilibrium populations of zero field, then the $I=7 / 2$ resonance (a former ground state) will have the strongest intensity, not the weakest. We deduce that, in contrast to higher temperatures where the state populations are thermally equilibrated before the ac probe field is applied, at low temperature the system does not fully equilibrate in response to the changes in temperature and applied magnetic field that take place before the measurement. Yet it is clear that Eq. (3) is valid, regardless of whether or not the $p_{i}$ 's are Boltzmann populations, so observation of the isolated susceptibility can be used to measure how the actual $p_{i}$ 's depend on energy. Analyzing the details of this behavior theoretically is an interesting challenge that is beyond the scope of this paper. We also remark that there is potential for the control of nonequilibrium state populations by pumping and then subsequent measurement by susceptometry.

\section{CONCLUSION}

In conclusion, although there has been much work on the susceptibility of isolated rare-earth ions, including detailed master-equation-based analyses of experimental data [21-25], it appears that a simple reduction to a well-defined isolated susceptibility has not been previously observed. Our observation of it in dilute spin ice has been enabled by the unique local environment of the $\mathrm{Ho}^{3+}$ ion, which allows a very simple effective Hamiltonian [Eq. (13)] to be enacted. Effects similar to those reported here should be observable for $\mathrm{Ho}^{3+}$ in other environments or for other non-Kramers rare-earth ions. However, variations in the spin Hamiltonian may shift the regime of observability of the isolated susceptibility outside the usual frequency window. For example, in the well-studied system $\mathrm{LiHo}_{x} \mathrm{Y}_{1-x} \mathrm{~F}_{4}$ [31], there is a low-lying crystal field level above the ground state doublet that will introduce further transverse terms into the spin Hamiltonian, assisting relaxation. This means that the new generation of ac susceptometers [3] that can measure into the megahertz range could be crucial to observe a purely isolated response.

In regard to the specific case of $\mathrm{Ho}^{3+}$ in the spin ice environment, it is worth comparing our results with the calculations of Tomasello et al. [32]. These authors used the full crystal field Hamiltonian, rather than making the effective spin-1/2 approximation used here, but they did not include hyperfine contributions or our phenomenological $\Delta$ terms that arise from random strains in practical samples. Use of the full crystal field Hamiltonian allows coupling of the effective spins to a transverse field, leading to level crossing resonances similar to those induced by $\Delta$, but at high transverse field values: typically of order $10 \mathrm{~T}$ and above. This justifies our neglect of $g_{\perp}$ in the present work. The $\chi^{z z}$ susceptibility calculated in Ref. [32] is equivalent to our $\chi_{T}$. Figure 8(a) of Ref. [32] shows that the calculated $\chi_{T}$ in zero field is in close agreement with our experimental measurement (our Fig. 4) in the equivalent temperature range, again justifying our neglect of higher crystal field states.

At a more conceptual level, our results highlight the difference between the processes imagined when one formulates the thermodynamic adiabatic and quantum adiabatic (isolated) susceptibilities [Eqs. (2) and (3)] to describe the response of the system to a change in applied field, $B \rightarrow B+d B$. In the thermodynamic case, the process is thermodynamically reversible: the state of the system in the field $B+d B$ is a thermal equilibrium state. In the quantum case the process is only mechanically reversible: the system is out of thermal equilibrium in the field $B+d B$. This begs the question, if state populations cannot respond to a change in field, how could a Boltzmann population be prepared in the first place? The answer is that one needs the equilibrium state in a field $B$ to be prepared on some timescale that is sufficiently long for equilibrium to be established but the perturbing field $d B$ to be applied on a timescale that is sufficiently short that changes in state population do not occur. Our experiments furnish 
examples that achieve this condition (here, at higher temperature) as well as examples that do not (here, at lower temperature). In the latter case our results indicate that a Boltzmann population cannot be prepared, and we would expect that the actual distribution (and hence response) will show a complicated history dependence: our initial measurements confirm this expectation, but fully characterizing this behavior will be a major project. Here, we confine ourselves to the conclusion that the isolated susceptibility may potentially be used to directly measure the nonequilibrium state populations. This indicates a promising avenue of research in the context of spin ice and other rare-earth magnets, as it suggests a way to test nonequilibrium theories, the concept of effective temperature [33], and so on.

Finally, we have shown how the isolated susceptibility is a direct measure of concurrence $\mathcal{C}$ between spin states, with $\mathcal{C}=1$ at the avoided level crossings. This infers that the apical spin population in our dilute spin ice sample shows complete, or nearly complete, concurrence at these special points. We are not aware of any other examples of an experimental measurement of state concurrence in a real magnetic system. Whether or not this ability to measure concurrence translates to more strongly interacting systems is an open question, but a strongly interacting system showing these effects may be afforded by bulk (concentrated) spin ice, where spin flipping associated with "monopole" excitations gives similar peaks in the high-frequency susceptibility [34,35].

\section{ACKNOWLEDGMENTS}

S.R.G. and S.T.B. would like to thank EPSRC for funding, Grants No. EP/S016465/1 and No. EP/S016554/1, respectively. E.L. and C.P. acknowledge financial support from ANR, France, Grant No. ANR-15-CE30-0004. S.R.G. would also like to thank A. Armour for illuminating discussions.

\section{APPENDIX: DERIVATION OF EQUATIONS (1)-(3)}

We define $m$ as the magnetic moment of the system and assume thermodynamic equilibrium, so that $m$ is parallel to the applied magnetic field $H=B / \mu_{0}$ and can be treated as a scalar. The magnetic moment is given rather generally by $m=\operatorname{Tr}[\rho \hat{m}]$, where $\rho$ is the (normalized) density operator and $\hat{m}$ is the magnetic moment operator, but in terms of eigenstates $i$ and energy eigenvalues $E_{i}$, it can be more conveniently written: $m=\sum_{i} m_{i} p_{i}$, where $m_{i}=-\partial E_{i} / \partial B$ is the magnetic moment of each state and $p_{i}$ is the Boltzmann probability: $p_{i}=\frac{e^{-E_{i} / k_{\mathrm{B}} T}}{\sum_{i} e^{-E_{i} / k_{\mathrm{B}} T}}$. The magnetization is the magnetic moment per unit volume, and the susceptibility is the gradient of this with respect to applied field. Performing the differentiation gives

$$
\chi=\frac{\partial M}{\partial H}=\frac{1}{V}\left[\sum_{i} m_{i}\left(\partial p_{i} / \partial H\right)+\sum_{i}\left(\partial m_{i} / \partial H\right) p_{i}\right]
$$

The three susceptibilities in Eqs. (1)-(3) in the main text are determined by the constraints applied as the field is adjusted such that $H \rightarrow H+d H$. The isothermal definition, Eq. (1), assumes that $p_{i} \rightarrow p_{i}+d p_{i}$, which are the equilibrium probabilities at temperature $T$ in applied field $H+d H$. Hence, $\chi_{T}$ is composed of both terms in the square brackets of the above expression. The quantum adiabatic or isolated susceptibility, Eq. (3), in contrast assumes that $d p_{i}=0$, so only the second term survives. In terms of thermal (canonical) averages $\langle\cdots\rangle$ at fixed $T$ the difference may be expressed as

$$
\chi_{T}=d\langle M\rangle / d H, \quad \chi_{I}=\langle d M / d H\rangle .
$$

Finally, the thermodynamic adiabatic susceptibility assumes that $d p_{i}$ are changes in equilibrium probabilities reached by holding the entropy, rather than the temperature, constant. The thermodynamic relationship (2) is derived as follows. We write $M=M(H, T)$ so that

$$
d M=\left(\frac{\partial M}{\partial H}\right)_{T} d H+\left(\frac{\partial M}{\partial T}\right)_{H} d T
$$

and that

$$
\left(\frac{\partial M}{\partial H}\right)_{S}=\left(\frac{\partial M}{\partial H}\right)_{T}+\left(\frac{\partial M}{\partial T}\right)_{H}\left(\frac{\partial T}{\partial H}\right)_{S} .
$$

To obtain Eq. (2), we then note that the first two terms in Eq. (A4) are $\chi_{S}$ and $\chi_{T}$, respectively, and that the third term may be rearranged with the help of the following three relations:

$$
\begin{gathered}
(\partial T / \partial H)_{S}=-\frac{(\partial S / \partial H)_{T}}{(\partial S / \partial T)_{H}}, \\
(\partial S / \partial H)_{T}=\mu_{0} V(\partial M / \partial T)_{H}, \\
(\partial S / \partial T)_{H}=C_{H} / T .
\end{gathered}
$$

The first of these relations is an identity of partial derivatives, the second is a Maxwell relation derived from the fundamental equation $d U=T d S+\mu_{0} V H d M$, and the third is easily derived from the latter equation via a Legendre transform to the magnetic enthalpy $\mathcal{E}=U-\mu_{0} V M H$. The adiabatic susceptibility can also be expressed in terms of averages over the energies $E_{i}$ and their field derivatives (see Ref. [8]), but we retain the thermodynamic definition here to emphasize that $\chi_{S}$ does not contain any information that is not already contained in measurements of the magnetization and specific heat.
[1] H. B. G. Casimir and F. K. du Pré, Physica (Amsterdam) 5, 507 (1938).

[2] C. V. Topping and S. J. Blundell, J. Phys.: Condens. Matter 31, 013001 (2019).

[3] E. Riordan, J. Blomgren, C. Jonasson, F. Ahrentorp, C. Johansson, D. Margineda, A. Elfassi, S. Michel, F. Dell'ova, G. M. Klemencic, and S. R. Giblin, Rev. Sci. Instrum. 90, 073908 (2019).
[4] L. J. F. Broer, Physica (Amsterdam) 17, 531 (1951).

[5] R. M. Wilcox, Phys. Rev. 174, 624 (1968).

[6] R. Pirc and B. G. Dick, Phys. Rev. B 9, 2701 (1974).

[7] Except, perhaps, by Amaya et al. in the context of cross relaxation. K. Amaya, Y. Tokunaga, Y. Kuramitsu, and T. Haseda, J. Phys. Soc. Jpn. 33, 49 (1972).

[8] Moriya has drawn a very similar figure; see T. Moriya, Busseiron Kenkyu 3, 332 (1958), Fig. 6. 
[9] L. D. Landau and E. M. Lifshitz, Quantum Mechanics, 3rd ed. (1977), p. 148.

[10] J. R. Rubbmark, M. M. Kash, M. G. Littman, and D. Kleppner, Phys. Rev. A 23, 3107 (1981).

[11] F. Troiani, C. Godfrin, S. Thiele, F. Balestro, W. Wernsdorfer, S. Klyatskaya, M. Ruben, and M. Affronte, Phys. Rev. Lett. 118, 257701 (2017).

[12] M. N. Leuenberger and D. Loss, Phys. Rev. B 61, 1286 (2000).

[13] R. Kubo, J. Phys. Soc. Jpn. 12, 570 (1957).

[14] W. Marshall and R. D. Lowde, Rep. Prog. Phys. 31, 705 (1968).

[15] One also recognizes in Eq. (4) the second-order term in Van Vleck's susceptibility equation: for idealized Van Vleck (singlet ground state) paramagnets, in which only this term is retained by construction, $\chi_{T}$ becomes trivially equal to $\chi_{\mathrm{I}}$ because changes in thermal populations are neglected.

[16] W. K. Wootters, Phys. Rev. Lett. 80, 2245 (1998).

[17] A. Abragam and B. Bleaney, Electron Paramagnetic Resonance of Transition Ions (Oxford University Press, Oxford, 1970).

[18] S. Rosenkranz, A. P. Ramirez, A. Hayashi, R. J. Cava, R. Siddharthan, and B. S. Shastry, J. Appl. Phys. 87, 5914 (2000).

[19] Because the time-reversal symmetry of the ground doublet is not protected by Kramers's theorem, low-symmetry crystal electric fields induced by lattice strains can generally act to remove it.

[20] S. Hüfner and G. Weber, Phys. Lett. 13, 115 (1964).

[21] R. Giraud, A. M. Tkachuk, and B. Barbara, Phys. Rev. Lett. 91, 257204 (2003).
[22] S. Bertaina, B. Barbara, R. Giraud, B. Z. Malkin, M. V. Vanuynin, A. I. Pominov, A. L. Stolov, and A. M. Tkachuk, Phys. Rev. B 74, 184421 (2006).

[23] M. J. Graf, J. Lago, A. Lascialfari, A. Amato, C. Baines, S. R. Giblin, J. S. Lord, A. M. Tkachuk, and B. Barbara, Phys. Rev. Lett. 99, 267203 (2007).

[24] B. Barbara, V. Zapasskii, G. Kozlov, B. Malkin, M. Vanyunin, and V. Reiterov, Opt. Spectros. 104, 218 (2008).

[25] R. C. Johnson, B. Z. Malkin, J. S. Lord, S. R. Giblin, A. Amato, C. Baines, A. Lascialfari, B. Barbara, and M. J. Graf, Phys. Rev. B 86, 014427 (2012).

[26] D. Prabhakaran and A. Boothroyd, J. Cryst. Growth 318, 1053 (2011).

[27] C. Paulsen, Introduction to Physical Techniques in Molecular Magnetism: Structural and Macroscopic Techniques, edited by F. Palacio, E. Ressouche, and J. Schweizer (Servicio de Publicaciones de la Universidad de Zaragoza, Zaragoza, Spain, 2001).

[28] L. Bovo, L. D. C. Jaubert, P. C. W. Holdsworth, and S. T. Bramwell, J. Phys.: Condens. Matter 25, 386002 (2013).

[29] A. M. Stoneham, Rev. Mod. Phys. 41, 82 (1969).

[30] J. Snyder, B. G. Ueland, J. S. Slusky, H. Karunadasa, R. J. Cava, and P. Schiffer, Phys. Rev. B 69, 064414 (2004).

[31] R. Giraud, W. Wernsdorfer, A. M. Tkachuk, D. Mailly, and B. Barbara, Phys. Rev. Lett. 87, 057203 (2001).

[32] B. Tomasello, C. Castelnovo, R. Moessner, and J. Quintanilla, Phys. Rev. B 92, 155120 (2015).

[33] L. F. Cugliandolo, J. Phys. A 44, 483001 (2011).

[34] L. Bovo, J. A. Bloxsom, D. Prabhakaran, G. Aeppli, and S. T. Bramwell, Nat. Commun. 4, 1 (2013).

[35] M. J. Matthews, C. Castelnovo, R. Moessner, S. A. Grigera, D. Prabhakaran, and P. Schiffer, Phys. Rev. B 86, 214419 (2012). 